\title{
Registro de mamíferos fósseis da Lapa do Bode, Ituaçu, Bahia
}

Record of fossil mammals of Lapa do Bode, Ituaçu, Bahia

\author{
R. A. Leoni ${ }^{*}$; L. A. Silva ${ }^{2}$ \\ ${ }^{1}$ Laboratório de Ecologia e Geociências, Universidade Federal da Bahia/IMS/CAT, 45029094, Vitória da \\ Conquista-Bahia, Brasil \\ ${ }^{2}$ Programa de Pós-graduação em Genética, Biodiversidade e Conservação, Universidade Estadual do Sudoeste da \\ Bahia, 45205-409, Jequié-Bahia, Brasil
}

*ronaldoaleoni@gmail.com

(Recebido em 30 de setembro de 2018; aceito em 29 de novembro de 2018)

O presente trabalho realizou a identificação taxonômica de mamíferos fósseis de cinco taxa pertencentes aos gêneros: Alouatta, Myocastor, Tamandua, Pecari e Tapirus, encontrados na gruta Lapa do Bode localizada no município de Ituaçu-BA. Os fósseis foram transportados e triados no Laboratório de Ecologia e Geociências localizado na Universidade Federal da Bahia, Campus Anísio Teixeira, Instituto Multidisciplinar em Saúde, Vitória da Conquista. Os táxons foram identificados em sua maioria por uso da fórmula dentária, características morfometrias e morfológicas descritas na literatura e/ou comparação com espécimes da coleção do Laboratório. Os materiais variaram em estado de preservação, logo o conjunto faunístico pode não representar uma única comunidade. Estudos futuros poderão corroborar a hipótese apresentada.

Palavras-chave: Fósseis, cavernas, mamíferos.

This paper executed the taxonomic identification of mammals fossils belonging to five different genders: Alouatta, Myocastor, Tamandua, Pecari and Tapirus, found in gruta Lapa do Bode localized in the city of Ituaçu-BA, they were transported and treated in Laboratório de Ecologia e Geociências located in Universidade Federal da Bahia, Campus Anísio Teixeira, Instituto Multidisciplinar em Saúde, Vitória da Conquista. The taxa were mostly identified by use of the dental formula, morphometric and morphological characteristics described in the literature and/or comparison with specimens from the Laboratory collection. The materials varied in a state of preservation, so the faunistic set may not represent a single community. Future studies may corroborate the hypothesis presented.

Keywords: Fossils, caves, mammals.

\section{INTRODUÇ̃̃o}

As cavernas, também conhecidas como grutas, furnas, buracos e tocas, são cavidades naturais subterrâneas, com espaços singulares que podem abrigar grande complexidade de formações geológicas, ecossistemas únicos e bastante sensíveis, bem como importantes achados arqueológicos, paleobiológicos e paleontológicos [1]. Devido ao alto grau de preservação dos fósseis em cavernas, esses ambientes tornam-se um local de grande interesse para estudos paleontológicos e paleoecológicos.

Os animais podem entrar nesses locais em busca de abrigo ou água, ou seus ossos podem ser transportados por rios submersos e/ou por inundações periódicas. Uma vez dentro da caverna, esses remanescentes são protegidos da ação do sol, vento ou chuva e da ação de predadores, resultando em preservação por vários processos de fossilização [2].

Em todas as regiões geográficas brasileiras existem registros de mamíferos fósseis, sendo a maioria do período pleistocênico, com representantes de várias ordens, como: Rodentia, Chiroptera, Marsupialia, Xenarthra, Carnivora, Notoungulata, Cetacea, Artiodactyla, Primates, Litopterna, Condylarthra, Perissodactyla, Sirenia, Astrapotheria, Lagomorpha, Xenungulata, Pyrotheria, Proboscidea [3]. Segundo Bergqvist (2004) [3] foram representadas 77 famílias, sendo os roedores os mais diversos, e o grupo Xenarthra com o dobro de grupos dos existentes atualmente. 
O Estado da Bahia abriga, em seus ambientes deposicionais como tanques e cavernas, diversos fósseis, sobretudo de mamíferos do Quaternário, tornando-se um estado com um alto potencial fossilífero [4]. Lessa et al. (1998) [5], registraram a ocorrência de diversas espécies de mamíferos nas cavernas Toca da Boa Vista, Toca dos Ossos, Gruta dos Brejões e Grutas de Iraquara, como Leopardus, Puma, Panthera, Cerdocyon e Conepatus. Em adição ao conhecimento da fauna pleistocênica, segundo esses autores a composição da fauna de mamíferos carnívoros no Pleistoceno Final e início do Holoceno teve uma taxa específica e muito mais representativa do que a atual, em função das condições climáticas.

Ainda pra o estado da Bahia, já foram encontrados mamíferos fósseis de taxa viventes pertencentes aos gêneros Alouatta, na caverna Toca do Clovis Saback I [6], Coendou, Panthera e Tamandua, na Toca da Barriguda [7], Didelphis, Gracilinanus, Incertae, Marmosa, Marmosops, Metachirus e Monodelphis [8], na Toca da Boa Vista, todas localizadas no município de Campo Formoso. Na divisa dos municípios de Iuiú e Malhada, nas cavernas Toca Fria e Jatobá, foram registrados a ocorrência dos gêneros Tapirus, Tayassu e Mazama [9].

Este trabalho teve como objetivo registrar a ocorrência de fósseis de mamíferos provenientes da caverna Lapa do Bode, Bahia, Brasil, bem como ampliar o conhecimento da história natural da gruta.

\title{
2. MATERIAL E MÉTODOS
}

As coletas foram realizadas na Lapa do Bode $\left(13^{\circ} 50^{\prime} 11^{\prime \prime} \mathrm{S} ; 41^{\circ} 17^{\prime} 07^{\prime \prime} \mathrm{W}\right)$ localizada a sudeste da zona urbana de Ituaçu, Bahia, em dezembro de 2017 e março de 2018. Fazendo parte da Serra do Sincorá, Chapada Diamantina Meridional [10], a Lapa do Bode é a maior caverna conhecida no relevo cárstico da bacia do rio Una, com aproximadamente $5,3 \mathrm{~km}$ de desenvolvimento planimétrico [11]. O material foi coletado em três corredores distintos da caverna, a maioria com pouca incrustação.

Os fósseis foram triados e identificados até o menor nível taxonômico e tombados na coleção paleontológica do Laboratório de Ecologia e Geociências (LEG) do Instituto Multidisciplinar em Saúde da Universidade Federal da Bahia, campus Anísio Teixeira (IMS/CAT-UFBA), Vitória da Conquista, Bahia. Para a identificação foram utilizadas bibliografias específicas para cada táxon, bem como comparação do material com os já existentes na coleção do LEG.

\section{RESULTADOS E DISCUSSÃO}

\subsection{PALEONTOLOGIA SISTEMÁtica}

\author{
Ordem PRIMATES Linnaeus, 1758 \\ Subordem HAPLORRHINI Pocock, 1918 \\ Infraordem SIMIIFORMES Haeckel, 1866 \\ Família ATELIDAE Gray, 1825 \\ Subfamília ALOUATTINAE Trouessart, 1897
}

Alouatta Lacépède, 1799

Alouatta sp.

Material: Fragmento da maxila esquerda (LEG1531).

Descrição: No fragmento da maxila esquerda (Figura 1A) estão presentes parte do canino (C1), três pré-molares (P1, P2 e P3) e primeiro e segundo molar (M1 e M2). A morfologia da maxila LEG1531 se assemelha com a descrita para o gênero Alouatta, apresentando a fórmula dentária I2/2, C1/1, P3/3, M3/3, totalizando 36 dentes [12]. Comparando o comprimento da fileira superior de dentes (CDS) com as medidas apresentadas por Gregorin (2006) [13] e Araújo et al. (2016) [6] (Tabela 1), nota-se que as medidas assemelham-se com diversas espécies do gênero e são menores 
que as encontradas para machos, sugerimos assim que a maxila pertencia a uma fêmea, já que esse gênero apresenta uma dimorfismo sexual evidente [13].

Comentário: Estes primatas, popularmente conhecidos como bugios ou guaribas, têm hábito arbóreo, usando todos os níveis de altura da floresta, mas de forma mais frequente utilizam o dossel e emergências, os indivíduos desse gênero possuem características de locomoção distintas como uma lenta progressão de modo quadrúpede. Além do movimento entre as árvores esses primatas também fazem o movimento pelo chão, de modo cauteloso e furtivo, podendo ser facilmente superados em velocidade por humanos, em áreas de florestas descontínuas eles se movem através de grandes espaços entre árvores [14].

Esse gênero é amplamente distribuído geograficamente, preferem florestas primárias, porém são altamente adaptáveis e podem ser encontrados em vários tipos de ambientes, como em pântanos, florestas decíduas, mangues, sempre viva e florestas ripárias [14].

A distribuição atual do gênero Alouatta que mais se aproxima da área de estudo inclui a espécie A. belzebul, na divisa dos estados de Alagoas, Bahia e Pernambuco, e A. guariba (A. clamitans), ao sul da Bahia [15].

O gênero Alouatta é o mais estudado dos macacos do Novo Mundo, mas apesar disso ainda apresenta uma classificação taxonômica não completamente definida [16]. Como o material encontrado é considerado insuficiente para uma atribuição específica, a classificação manteve-se apenas em nível de gênero.

Tabela 1: Medidas (em mm) da maxila (LEG1531), comparados com outras espécies do gênero (Gregorin, 2006; Araújo et al., 2016). CDS - comprimento da fileira superior de dentes; F-Feminino; M-Masculino.

\begin{tabular}{ccc}
\hline Espécies do gênero Alouatta & \multicolumn{2}{c}{ CDS (mm) } \\
\hline LEG 1532 & \multicolumn{2}{c}{33.3} \\
Alouatta sp. (LEG 0314) & \multicolumn{2}{c}{29.0} \\
\cline { 2 - 3 } & \multicolumn{2}{c}{ Sexo } \\
\cline { 2 - 3 } A caraya & 35.8 & 42.2 \\
A. fusca & 35.6 & 40.5 \\
A. guariba (A. clamitans) & 36.1 & 40.4 \\
A.discolor & 35.3 & 40.88 \\
A.ululata & 35.0 & 41.8 \\
A.belzebul & 35.0 & 40.5 \\
A.nigerrima & 37.6 & 43.1 \\
A. macconnelli & 36.6 & 42.6 \\
A.juara & 36.7 & 43.8 \\
\hline
\end{tabular}

Ordem RODENTIA Bowdich, 1821

Subordem HYSTRICOMORPHA Brandt, 1855

Infraordem HYSTRICOGNATHI Brandt, 1855

Família MYOCASTORIDAE Ameghino, 1902

Myocastor Kerr, 1792

Myocastor coypus Molina, 1782

Material: Fragmento do fêmur (LEG1534).

Descrição: O fóssil de Myocastor foi encontrado em ótimo estado, porém inteiramente incrustado, impossibilitando a retirada dos remanescentes do local. No entanto, em campo foram coletas as informações do crânio, com enfoque na mandíbula e dentário, para confirmação da 
identificação. No dentário é possível visualizar claramente o padrão inclinado na fileira de dentes, e a característica hipsodonte dos pré-molares e molares. O gênero Myocastor apresenta molares e pré-molares extremamente hipsodontes (com grande desenvolvimento da coroa), sempre enraizados, além disso, em suas fileiras de dentes, que convergem anteriormente e são inclinados, os molares superiores são marcadamente menor em tamanho, anteriormente. O padrão das dobras reentra, formando uma dobra para dentro no molar superior, o tornando modificado, com dobras labiais e duas dobras linguais que se tornam lagos (fossetas) com desgaste. Os incisivos são amplos, com pigmento alaranjado nas superfícies anteriores [17].

Comentário: As espécies pertencentes ao gênero Myocastor são roedores de hábito aquático, herbívoros, além disso desfrutam de uma alta tolerância a condições climáticas e hidrológicas altamente adaptáveis [18]. Essa alta tolerância permitiu colonizam rapidamente habitats desocupados, por essa razão são também invasores de habitat de grande sucesso, sendo ausentes em distribuição somente na Austrália e na Antártica, são nativos da América do Sul, da sub-região patagônicas, ocorrendo na Argentina, Brasil, Bolívia, Paraguai, Uruguai e Chile. Se distribuindo por climas principalmente tropicais, subtropicais e temperados [19].

Esse gênero detém de uma distribuição geográfica natural na América do Sul restrita a territórios sub-tropicais: Argentina, Uruguai, Paraguai, Chile e Brasil. M. coypus foi registrado como fóssil por Lund (1887) em Lagoa Santa, Minas Gerais e por Cartelle em Jacobina, Bahia [20].

Ordem PILOSA Flower, 1883

Subordem VERMILINGUA Illiger, 1811

Família Myrmecophagidae Gray, 1825

Tamandua tetradactyla Linnaeus, 1758

Material: Crânio (LEG 1533), úmero esquerdo (LEG 1535), úmero direito (LEG 1536), fêmur direito (LEG 1537), fêmur esquerdo (LEG 1538), ulna direita (LEG 1539), tíbia esquerda (LEG 1540), tíbia direita (LEG 1541), rádio esquerdo (LEG 1542), ulna esquerda (LEG 1543), rádio direito (LEG 1544), escápula direita (LEG 1545), escápula esquerda (LEG 1546), fragmentos de costela (LEG 1547 até LEG 1566), vértebras (LEG 1567 até 1596, LEG 1626 até 1632, LEG 1612, LEG 1614, LEG 1616 até 1619), corpo de vértebra (LEG 1597), vértebras sacrais (LEG 1598), calcâneo direito (1602), calcâneo esquerdo (1603) e falange III (LEG 1622, LEG 1623).

Descrição: Crânio (LEG 1533) (Figura 1B-D) encontra-se quase completo. Entre as peças da região facial, está faltando a mandíbula, e porção distal da pré-maxila e o lado direito sobre a região lacrimal estão quebrados. A região esquerda do lacrimal apesar de presente encontra-se danificada e apenas o jugal direito está presente. As regiões nasal, maxilar e porção frontal estão preservadas. Das peças da região cerebral o côndilo occipital, osso parietal e supraoccipital foram preservados. Os Vermilingua dispõem de inúmeras sinapomorfias, incluindo uma curvatura do eixo basicranial/basifacial, uma exposição orbital maxilar reduzida, e o tubo eustáquio possui uma orientação posteromedial. Outras especializações no crânio são relacionadas ao uso da língua, a característica da qual o grupo deriva como o meio primário de captura de presas. Estes incluem um alongamento do rostro e do palato duro e a completa perda dos dentes. $\mathrm{O}$ arco zigomático é incompleto e o jugal é reduzido para um pequeno processo ligado ao processo zigomático da maxila. O palato duro é alongado como o resultado do leve alongamento dos palatinos e da junção dos pterigóides com a linha média, de modo que as narinas internas são deslocadas posteriormente para as fossas glenóides. O palato secundário alongado permite este gênero acomodar a língua retrátil com a orofaringe sem interferir com a habilidade de respirar [21].

O material coletado além de apresentar as características citadas acima, após medições e comparações morfométricas com exemplares disponíveis de Tamandua tetradactyla na coleção do Laboratório de Ecologia e Geociências, IMS/CAT-UFBA, mostrou-se morfologicamente e morfometricamente semelhantes como foi evidenciado na tabela (Tabela 2). 

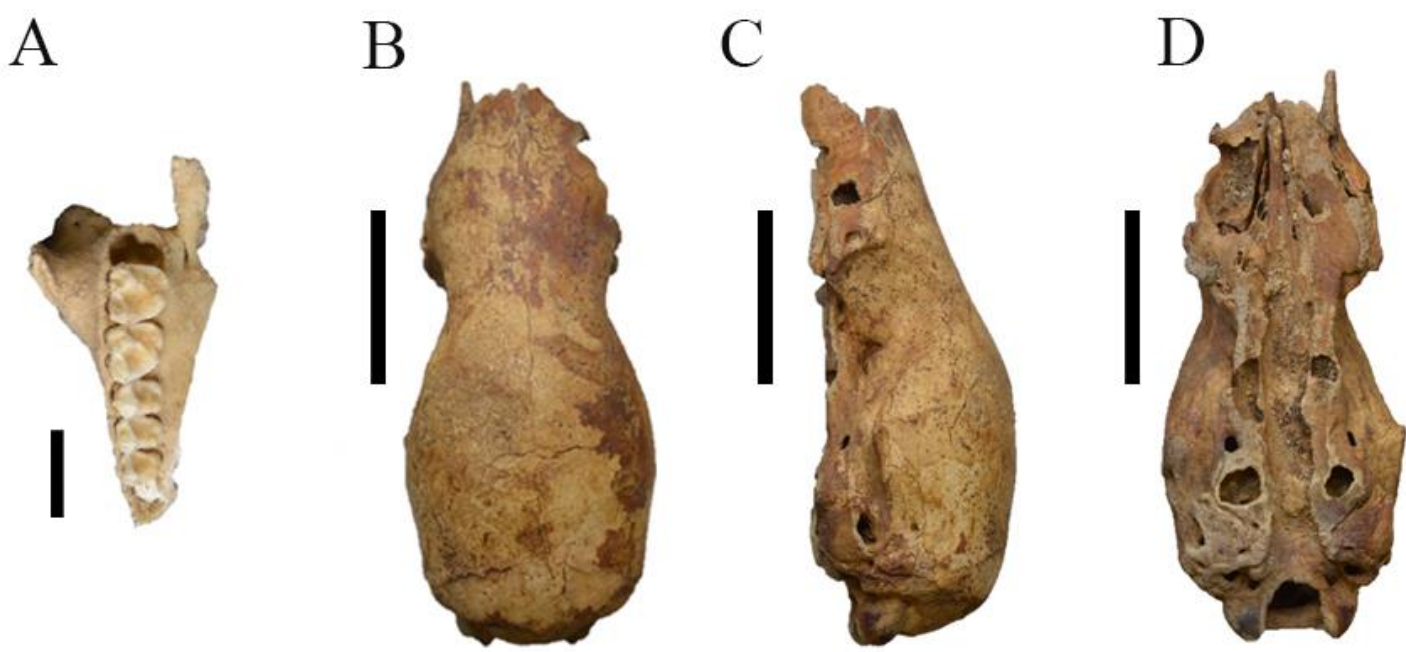

Figura 1: Fragmento da maxila esquerda de Alouatta sp.; escala: $1 \mathrm{~cm}$ (A); Crânio de Tamandua tetradactyla, visão dorsal (B) visão lateral (C) visão ventral (D); escala: $2 \mathrm{~cm}$.

Comentário: O gênero Tamandua apresenta uma morfologia altamente adaptada para o consumo de formigas e cupins, possuindo caracteres como fortes pernas dianteiras equipadas com poderosas garras, uma língua, longa, pegajosa e portátil, uma boca pequena, um focinho comprido e glândulas salivares bem desenvolvidas, como também um alto e refinado olfato. Tamanduás são geralmente solitários, mas podem ser encontrados em pares durante a época de acasalamento ou quando ainda estão carregando o filhote. Ainda há uma escassez de dados sobre padrões do movimento e área de vida, uma estimativa teórica da área de vida dos tamanduás baseadas somente nas características ecológicas concluiu que seu "home range" deveria ser de ao menos 9 $\mathrm{km}^{2}[22]$.

Tamandua tetradactyla é endêmica da América cisandina, tendo registros em todos os países da região. No Brasil a espécie ocorre em todos os biomas: Amazônia, Caatinga, Cerrado, Mata Atlântica, Pantanal e Pampa [23].

Tabela 2: Medidas (em mm) do crânio de Tamandua tetradactyla (LEG1533), comparado com LEG0641 (Crânio da Toca da barriguda) e LEG1183 (Crânio da Gruta da Marota).

\begin{tabular}{cccc}
\hline & LEG1533 & LEG0641 & LEG1183 \\
\hline Região Facial & & & \\
\hline Largura do rostro & 33.86 & 31.70 & 19.22 \\
Largura entre narinas & 26.52 & 21.58 & 24.62 \\
\hline Região Cranial & & & \\
\hline Largura da caixa craniana & 42.74 & 39.40 & 40.60 \\
Altura da caixa craniana & 30.50 & 22.00 & 28.00 \\
Largura do conduto auditivo esquerdo & 05.00 & 05.88 & 06.90 \\
Largura do conduto auditivo direito & 04.88 & 04.32 & 05.82 \\
Comprimento occipital nasal & 96.70 & 86.68 & 111.82 \\
Foramen magnum & 23.44 & 26.88 & 24.44 \\
\hline
\end{tabular}




\section{Ordem PERISSODACTYLA Owen, 1848 \\ Família TAPIRIDAE Gray, 1821}

\section{Tapirus terrestris Linnaeus, 1758}

Material: Dentário direito (LEG1523) e esquerdo (LEG1525) e fragmento de maxila (LEG1524).

Descrição: Dentário direito (Figura 2A e D) bem preservado, pertencente a um tapirídeo jovem apenas com dentes de leite, apresentando dm2 e dm3 emergidos e dm4 não emergido. Corpo, côndilo, região sinfisária, ângulo, alveolar e processo coronóide presentes. Além disso, a peça apresenta sinais claros de intemperismo e incrustação. O dentário esquerdo (Figura 2B e C), assim como o direito encontra-se bem preservado, com quatro dentes presentes, mas apenas $\mathrm{dm} 3$ totalmente emergido e o dm4 ainda não emergido. Corpo, côndilo, região sinfisária, ângulo, alveolar, processo coronóide presentes. Também apresenta sinais claros de intemperismo e incrustação.

O gênero Tapirus pôde ser identificado a partir da seguinte fórmula dentária: 3I/3i, 1C/1c, $4 \mathrm{P} / 3 \mathrm{p}$ e $3 \mathrm{M} / 3 \mathrm{~m}$, totalizando 42 dentes. Os incisivos e caninos são separados do $\mathrm{P} 1 / \mathrm{p} 2$ por um diastema [24]. Nos LEG1523 e LEG1525 estavam presentes a maioria dos dentes. Rancy (1981) [25] descreveu um indivíduo jovem detalhando a fórmula dentária e sua morfologia, características essas que permitiram identificar o indivíduo coletado como um jovem do gênero Tapirus.

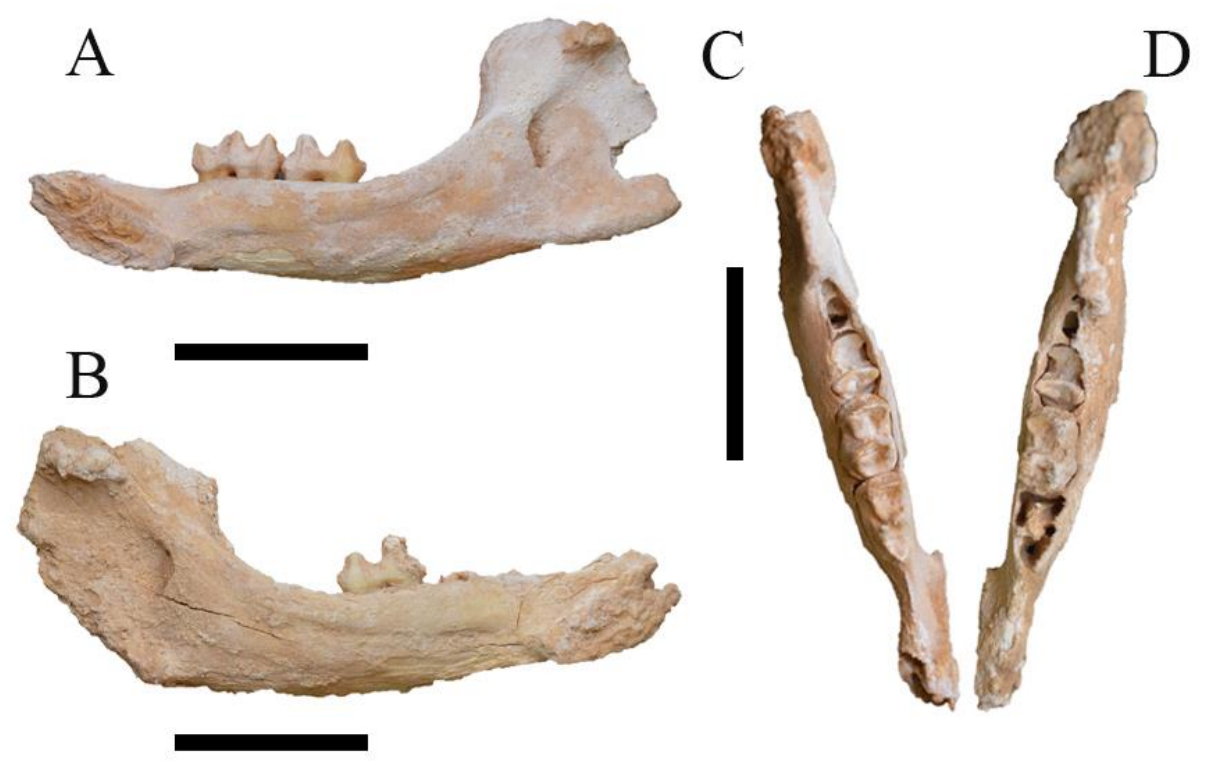

Figura 2: Visão lateral do dentário direito $(A)$ e esquerdo $(B)$ e visão oclusal do dentário direito $(C) e$ esquerdo (D) de Tapirus terrestris; escala $5 \mathrm{~cm}$.

Comentário: As antas permanecem inativas e descansam durante o dia e durante a noite saem para se alimentar. Apesar da sua dieta ser, de forma geral, composta de folhas e fibras, frutos são um importante componente da sua dieta. Um estudo na Amazônia mostrou que a dieta das antas desse local era composta de frutos com uma porcentagem de 33\%, demonstrando uma importante função ecológica do grupo, a dispersão de sementes [25].

Tapirus terrestris encontra-se presente em vários ambientes, desde florestas de galeria e florestas tropicais a zonas sazonalmente inundáveis, porém, a anta é um animal que vive, de maneira geral, em ambientes florestados associados a fontes de água permanentes. O nível de associação com outros tipos de habitat varia bastante, no entanto, florestas ripárias são ambientes muito importantes para Tapirus, da mesma maneira também possuem uma relação íntima com florestas de palmeiras [26]. 
Ordem ARTIODACTYLA, Owen, 1848

Família TAYASSUIDAE Palmer, 1897

Pecari tajacu Linnaeus, 1758

Material: Fragmentos de maxila direita (LEG1528), fragmento da mandíbula esquerda (LEG1526 e LEG1529), mandíbula esquerda (LEG1530), pré-molar (LEG1527).

Descrição: Mandíbula esquerda levemente danificada, com cinco dentes presentes ( 3 pré-molares e 2 molares). Corpo alveolar e região sinfisária danificadas, perdendo assim a porção distal da mandíbula. Côndilo, ângulo, processo coronóide presentes. Maxila direita (Figura 3A-B) danificada, não possuindo nenhum dente anexado. Apenas partes do corpo e ângulo estão presentes. Região sinfisária, alveolar, côndilo e processo coronóide ausentes.
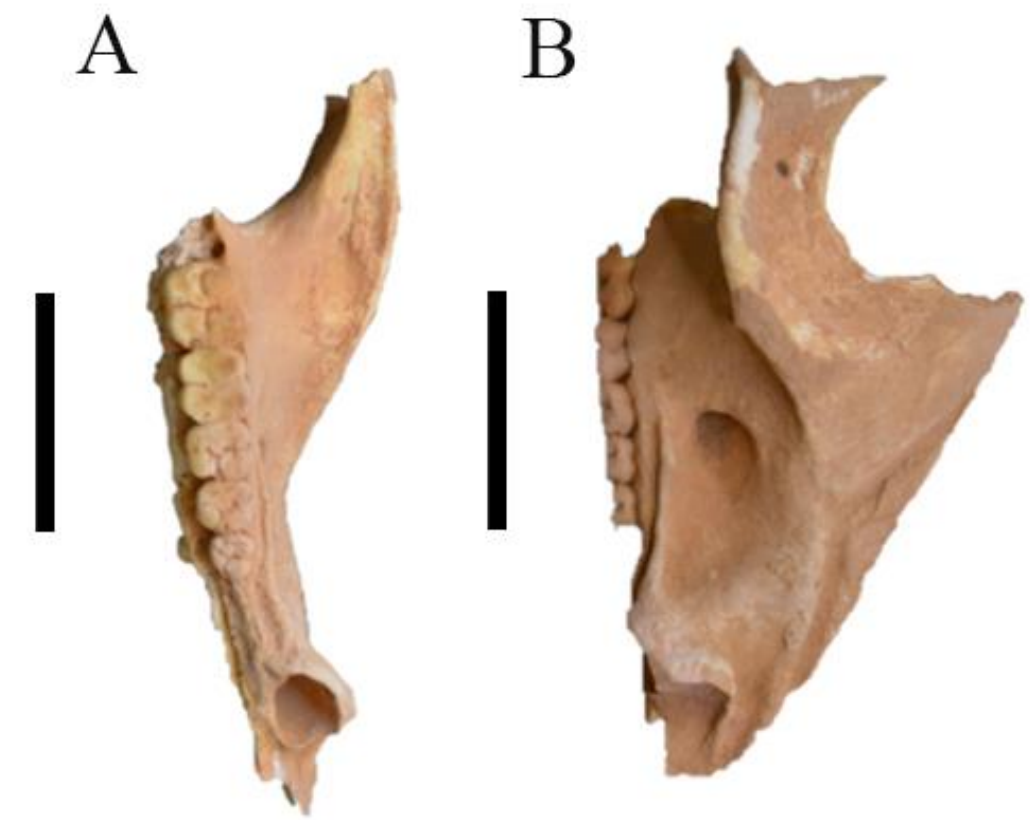

Figura 3: Fragmentos de mandíbula esquerda de Pecari tajacu vista superior (A) e visão lateral (B); escala: $2 \mathrm{~cm}$.

Comentário: O gênero Pecari pode ser reconhecido por algumas características dentárias, caracteres como a ausência de cíngulo basal nos molares, vestígio de cúspides acessórias entre as cúspides principais e o rostro mais largo do que o de outras espécies. Ainda podem ser observados P2 (Segundo Pré-Molar) de contorno triangular e P4 (Quarto Pré-Molar) molariforme com quatro cúspides distintas. O 1I (Primeiro Incisivo) possui um contorno ovalado e implanta-se de maneira oblíqua no alvéolo. O C (Canino) possui assim como o incisivo um formato ovalado e sua posição apical é orientada lateralmente. Os molares são levemente inflados e de aspecto bunodontes, sendo o M2 (Segundo Molar) o maior dente da série molar [27]. Os caninos dos Pecari tajacu são bastante desenvolvidos triangulares e direcionados para fora [28].

Sobre seu habitat, os $P$. tajacu são adaptados a uma grande diversidade de habitats, desde florestas tropicais úmidas a regiões semiáridas. Esta capacidade de sobrevivência da espécie em diferentes condições se faz graças a adaptações fisiológicas e comportamentais. No pantanal preferem as paisagens com predominância de floresta, mas também utilizam o cerrado. Seus bandos variam de 5 a 25 membros e dividem-se em subgrupos temporários que se unem no final da manhã e início da tarde, possuindo variação no número de membros em um grupo em estações chuvosas e secas na Amazônia. Esse grupo ainda é de suma importância na manutenção de ecossistemas como dispersores de sementes e predadores. Também são bioindicadores, por sua 
alta resistência a alterações no ambiente, geralmente são associados a habitats altamente alterados [29].

Os Pecari tajacu ocorrem em todas as regiões do Brasil e em todos os demais países sulamericanos, exceto Chile [28]. Os catetos têm ampla distribuição e resistência a alterações antrópicas, com exceção de ambientes altamente alterados. Os $P$. tajacu podem ainda serem encontrados nas áreas de cobertura vegetal de qualquer bioma estas áreas compreendem em $12 \%$ da área original da Mata Atlântica, 50\% do Cerrado, 30\% da Caatinga e 80\% da Amazônia e Pantanal [29].

\section{CONCLUSÃO}

Devido a possibilidade de alta mistura temporal, o conjunto faunístico encontrado na Lapa do Bode pode não representar uma única paleocomunidade do Pleistoceno Tardio - Holoceno. Futuros estudos poderão corroborar a hipótese apresentada.

\section{REFERÊNCIAS BIBLIOGRÁFICAS}

1. Monteiro FAD. A Espeleologia e as cavernas no Ceará: conhecimentos, proteção ambiental e panorama atual. [dissertação]. Fortaleza (CE): Universidade Federal do Ceará; 2014. 15 p.

2. Bonito RA, Ferreira R L, Marinho TS. Occurrence and analysis of borings made by solitary wasp in caves: a study in cave ichnology. Rev Bras Paleontol. 2011;14:93-102, doi:10.4072/rbp.2011.1.10

3. Bergqvist LP, Almeida EB. Biodiversidade de mamíferos fósseis brasileiros. Rev Geociências. 2013;9(6):54-68.

4. Dantas MAT, Zucon MH. Occurrence of Catonyx cuvieri (lund, 1839) (Tardigrada, Scelidotheriinae) in late Pleistocene-Holocene of Brazil. Rev Bras Paleont. 2007;10(2):129-132. doi:10.4072/rbp.2007.2.06

5. Lessa G, Cartelle C, Faria HD, Gonçalves PR. Novos achados de mamíferos carnívoros do Pleistoceno final - Holoceno em grutas calcárias do estado da Bahia. Acta Geol Leopoldensia. 1999;21(46-47):157169.

6. Araujo AV, Dantas MAT, Ximener CL. Registro de Alouatta sp. (Primates, Alouattinae) em depósito espeleológico do Quaternário do estado da Bahia. Espeleo-Tema. 2016;27(6).

7. Silva LA, Dantas MAT, Araújo AV. Registro e Análise Tafonômica de Pequenos e Médios Mamíferos em Cavernas na Bahia. In: ANAIS do $34^{\circ}$ Congresso Brasileiro de Espeleologia; 2017, Ouro Preto. Minas Gerais: Sociedade Brasileira de Espeleologia: 2017. p. 577.

8. Rosário B, Oliveira B, Kraemer B. Caracterização taxonômica de Didelphimorphia gill, 1872 da Toca da Boa Vista - BA, Brasil. In: Rasteiro MA, Sallun Filho W. (Eds.) Congresso Brasileiro De Espeleologia, 2015. Campinas (SP) Eldorado SBE, 2015. p.1-7.

9. Dantas MAT, Missagia R, Dutra RP, Raugust T, Silva LA, Delicio MP, Renó R. Identificação taxonômica dos fósseis de mamíferos da caverna Toca Fria e Jatobá, Iuiú, Bahia: inferências paleoecológicas e temporais. In: Rasteiro MA, Morato L. (Eds.) Congresso Brasileiro de Espeleologia, 2013. Barreiras (BA), SBE, 2013. p. 433-438.

10. Gôuvea EP. Reencontro e "ocorrência curiosa" de Trichodamon froesi Mello-Leitão (Arachnida, Amblypygi). Rev Bras Zool. 1993;10(3):383-388. doi:10.1590/S0101-81751993000300002

11. Karmann I, Pereira RGFA, Mendes LF. Poço Encantado, Chapada Diamantina (Itaetê), BA: caverna com lago subterrâneo de rara beleza e importância cientifica. In: Schobbenhaus C, Campos DA, Queiroz ET, Winge M, Berbert-Born MLC. (Editores.) Sítios Geológicos e Paleontológicos do Brasil. Brasilia (DF) DNPM/CPRM - Comissão Brasileira de Sítios Geológicos e Paleobiológicos (SIGEP); 2002. p. 491-498.

12. Gregorin R, Simões SAM, Lima IJ, Júnior JSS. Mamíferos do Brasil: Guia de Identificação. 1 ed. Rio de Janeiro (Rio de Janeiro): Technical Books Editora, 2010. Capítulo 5 Ordem Primates; p. 160

13. Gregorin R. Taxonomia e variação geográfica das espécies do gênero Alouatta Lacépède (Primates, Atelidae), no Brasil. Rev Bras Zool. 2006;23(1):64-144, doi:10.1590/S0101-81752006000100005

14. Neville MK, Glander K, Braza E, Rylands AB. Ecology and behavior of neotropical primates 1 ed Washington D.C (Washington): World Wildlife Fund, 1988. Chapter 6 The Howling Monkeys, genus Alouatta; p. 349-453 In: Mittermeir, R. A; Rylands, A. B; Coimbra-filho, A; Fonseca, G. A. B. (Org.). Washington; 1988. p. 349-453 
15. Cortés-Ortíz L, Bermingham E, Rico C.; Rodríguez-Luna E, Sampaio I, Ruizgarcía M. Molecular systematics and biogeography of the neotropical monkey genus, Alouatta. Molec Phylogenet Evol. 2003;26(1):64-81, doi:10.1016/S1055-7903(02)00308-1

16. Inglêz APD. Caracterização genética de Alouatta caraya (Primates, Atelidae) utilizando marcadores heterólogos do tipo microssatélites [Dissertação]. Brasilia (DF): Universidade de Brasília; 2006.

17. Woods CA, Contreras L, Willner-chapman G, Whidden HP. Myocastor coypus. Mammalian Species. 1992;398:1-8, doi:10.2307/3504182

18. Courtalon P, Bó RF, Spina F, Jiménez N, Cantil L, Fernandez R, Porini G. Reproductive ecology of coypu (Myocastor coypus Molina, 1782) in the Middle Delta of the Paraná River, Argentina. Braz J Biol. 2015;75(1):30-38, doi:10.1590/1519-6984.06813

19. Bueno C. Ocorrência de Myocastor coypus Molina, 1782 no Estado do Rio de Janeiro. Boletim Soc Bras Mastozool. 2013;66:9-11.

20. Cartelle C, Lessa G. Presença de Myocastor coypus (Molina, 1782), Rodentia, Myocastoridae, do Pleistoceno final - Holoceno, no centro-oeste da Bahia. In: Anais do XI Congresso Brasileiro de Paleontologia; 1989 Set; Curitiba, PR: Sociedade Brasileira de Paleontologia; 1989. p. 1.

21. Mcdonald HG, Vizcaíno SF, Bargo MS. Skeletal anatomy and fossil history of the vermilingua. Flórida: University Press of Florida; 2008. Chapter 6, The Biology of Xenarthra; p. 65-66.

22. Rodrigues FHG, Medri IM, De miranda GHB, Camilo-alves C, Mourão G. Anteater Behavior and Ecology. Flórida: University Press of Florida; 2008. Chapter 24, The Biology of Xenarthra; p. 257.

23. Ohana JAB, Bertassoni A, Miranda FR, Mourão GM, Miranda GHB, Costa JF, Silva KFM, Fariacorrêa MA, Belentani SCS. Avaliação do Risco de Extinção de Tamandua tetradactyla (Linnaeus, 1758) no Brasil. Brasília (DF): ICMBio; 2015. Capítulo 9, Avaliação do Risco de Extinção dos Xenartros Brasileiros; p. 111.

24. Holanda EC. Os Tapiridae (Mammalia, Perissodactyla) do Pleistoceno Superior do Estado de Rondônia, Brasil. [Dissertação] Porto Alegre (RS): Universidade Federal do Rio Grande do Sul; 2007.

25. Rancy A. Mamíferos Fósseis do Cenozóico do Alto Juruá - Acre. [Dissertação] Porto Alegre (RS): Universidade Federal do Rio Grande do Sul; 1981.

26. Medici EP, Flesher K, Beisiegel BM, Keuroghlian A, Desbiez ALJ, Gatti A, Pontes ARM, Campos CB, Tófoli CF, Junior EAM, Azevedo FC, Pinho GM, Cordeiro JLP, Junior TSS, Morais AA, Mangini PR, Rodrigues LF, Almeida LB. Avaliação de risco da Anta Brasileira Tapirus terrestris Linnaeus, 1758, no Brasil. Biodivers Bras. 2012;2(3):103-116.

27. Martins CM, Oliveira EV. Novo material craniano de Tayassu Fischer, 1814, da formação Touro Passo (Pleistoceno superior), Rio Grande do Sul. Biodivers Pampeana. 2003;1(1):24-34.

28. Tomas WM, Tiepolo LM, Duarte JMB. Mamíferos do Brasil: Guia de Identificação. 1 ed. Rio de Janeiro (Rio de Janeiro): Technical Books Editora; 2010. Capítulo 10, Ordem Artiodactyla; p. 496.

29. Desbiez ALJ, Keuroghlian A, Beiseiegel BM, Medici EP, Gatti A, Pontes ARM, Campos CB, Tófoli CF, Junior EAM, Azevedo FC, Pinho GM, Cordeiro JLP, Júnior TSS, Morais AA, Mangini PR, Flesher K, Rodrigues LF, Almeida LB. Avaliação do Risco de Extinção do Cateto Pecari tajacu Linnaeus, 1758, no Brasil. Biodivers Bras. 2012;2(3):74-83. 